\title{
A Revised Tikhonov Regularization Method for a Cauchy Problem of Two-Dimensional Heat Conduction Equation
}

\author{
Songshu Liu $\mathbb{D D}^{1}$ and Lixin Feng $\mathbb{D}^{2}$ \\ ${ }^{1}$ School of Mathematics and Statistics, Northeastern University at Qinhuangdao, Qinhuangdao 066004, China \\ ${ }^{2}$ School of Mathematical Sciences, Heilongjiang University, Harbin 150080, China \\ Correspondence should be addressed to Lixin Feng; fenglixin@hlju.edu.cn
}

Received 22 December 2017; Revised 15 April 2018; Accepted 23 April 2018; Published 28 May 2018

Academic Editor: Filippo de Monte

Copyright (c) 2018 Songshu Liu and Lixin Feng. This is an open access article distributed under the Creative Commons Attribution License, which permits unrestricted use, distribution, and reproduction in any medium, provided the original work is properly cited.

In this paper we investigate a Cauchy problem of two-dimensional (2D) heat conduction equation, which determines the internal surface temperature distribution from measured data at the fixed location. In general, this problem is ill-posed in the sense of Hadamard. We propose a revised Tikhonov regularization method to deal with this ill-posed problem and obtain the convergence estimate between the approximate solution and the exact one by choosing a suitable regularization parameter. A numerical example shows that the proposed method works well.

\section{Introduction}

In many industrial applications [1] one wishes to determine the temperature on the surface of a body, where the surface itself is inaccessible for measurements. This problem leads us to consider a Cauchy problem of heat conduction equation, which can be considered as a data completion problem that means to achieve the remaining information from boundary conditions for both the solution and its normal derivative on the boundary. This sort of problem occurs in a wide range of scientific and engineering areas [1] including manufacturing process control, metallurgy, chemical, aerospace and nuclear engineering, food science, and medical diagnostics.

Mathematically, the Cauchy problem of heat equation belongs to the class of problems called the ill-posed problems; i.e., small errors in the measured data can lead to large deviations in the estimated quantities. As a consequence, its solution does not satisfy the general requirement of existence, uniqueness, and stability under small changes to the input data. To overcome such difficulties, a variety of techniques for solving the Cauchy problem of heat equation have been proposed [2-10].

In this paper, we investigate a Cauchy problem of twodimensional (2D) heat conduction equation. We remark here that the Cauchy problem of one-dimensional heat equation has been well studied in the last few decades. Due to severe ill-posedness, however, much more difficulties exist to solve the Cauchy problem of heat conduction equation in the $2 \mathrm{D}$ case than in the $1 \mathrm{D}$ case.

To the knowledge of the authors, there are still few results on Cauchy problem of heat conduction equation in the $2 \mathrm{D}$ case. In 2007, Qian and Fu [11] applied Fourier method and modified equation method to solve problem 1 and prove some error estimates of Hölder type for the solution. In 2011, Li and Wang [12] used a simplified Tikhonov regularization method to treat it. Following the works of [11, 12], Zhao et al. [13] also treated this problem by a modified kernel method in 2015 .

In this article, we continue to consider the following Cauchy problem of 2D heat conduction equation.

Problem 1.

$$
\begin{aligned}
& u_{t}(x, y, t)=u_{x x}(x, y, t)+u_{y y}(x, y, t) \text {, } \\
& 0<x<1, y>0, t>0 \text {, } \\
& u(0, y, t)=g(y, t), \quad y \geq 0, t \geq 0,
\end{aligned}
$$


with corresponding measured data function $g^{\delta}(y, t)$,

$$
\begin{array}{ll}
u_{x}(0, y, t)=0, & y \geq 0, t \geq 0, \\
u(x, y, 0)=0, & 0 \leq x \leq 1, \quad y \geq 0, \\
u(x, 0, t)=0, & 0 \leq x \leq 1, t \geq 0 .
\end{array}
$$

We wish to determine the temperature $u(x, y, t)$ for $0<$ $x \leq 1$ from temperature measurement $g^{\delta}(y, t)$. We will adopt a revised Tikhonov regularization method to deal with this problem. This method was first introduced by Carasso in [14], and then the idea of this method has been successfully used for solving various types of ill-posed problems [15-18]. Under a priori selection rule for the regularization parameter, the convergence of the revised Tikhonov regularization method will also be given.

The paper is organized as follows. In Section 2 we formulate a Cauchy problem of $2 \mathrm{D}$ heat conduction equation. Section 3 is devoted to the convergence estimate for this method. A numerical example is tested in Section 4. Finally, the paper ends with a brief conclusion in Section 5.

\section{Mathematical Formulation of the Cauchy Problem of 2D Heat Conduction Equation}

In order to use the Fourier transform technique, we extend the functions $u(x, y, t), g(y, t), g^{\delta}(y, t)$ to the whole real $(y, t)$ plane by defining them to be zero everywhere in $\{(y, t), y<0, t<0\}$. We wish to determine the temperature $u(x, y, t) \in L^{2}\left(\mathbb{R}^{2}\right)$ for $0<x \leq 1$ from the temperature measurement $g^{\delta}(y, t) \in L^{2}\left(\mathbb{R}^{2}\right)$.

We also use the corresponding $L^{2}$-norm defined as

$$
\|g\|=\left(\int_{\mathbb{R}^{2}}|g(y, t)|^{2} d y d t\right)^{1 / 2} .
$$

We now could assume that the measured data function $g^{\delta}(y, t)$ satisfies

$$
\left\|g^{\delta}-g\right\| \leq \delta
$$

where the constant $\delta>0$ represents a bound on the measurement error. Assume that there exists a constant $E>0$ such that the following a priori bound exists:

$$
\|u(1, \cdot, \cdot)\| \leq E
$$

Let

$$
\widehat{g}(\xi, \eta)=\frac{1}{2 \pi} \int_{\mathbb{R}^{2}} g(y, t) e^{-i(\xi y+\eta t)} d y d t, \quad \xi, \eta \in \mathbb{R}
$$

be the Fourier transform of a function $g(y, t)$. Then

$$
g(y, t)=\frac{1}{2 \pi} \int_{\mathbb{R}^{2}} \hat{g}(\xi, \eta) e^{i(\xi y+\eta t)} d \xi d \eta
$$

Applying this transform to (1) with respect to $y$ and $t$, we obtain

$$
\widehat{u}_{x x}(x, \xi, \eta)=\left(i \eta+\xi^{2}\right) \widehat{u}(x, \xi, \eta),
$$

which is a second-order ordinary differential equation for fixed $\xi$ and $\eta$. Now using the boundary condition in the frequency domain, we can easily get

$$
\widehat{u}(x, \xi, \eta)=\cosh (\tau x) \hat{g}(\xi, \eta),
$$

and taking the inverse Fourier transform, the solution of problem 1 is

$$
u(x, y, t)=\frac{1}{2 \pi} \int_{\mathbb{R}^{2}} e^{i(\xi y+\eta t)} \widehat{g}(\xi, \eta) \cosh (\tau x) d \xi d \eta
$$

where $\tau$ is the principal value of $\sqrt{i \eta+\xi^{2}}$; i.e.,

$$
\begin{aligned}
\tau & =\sqrt{i \eta+\xi^{2}} \\
& =\sqrt{\frac{\sqrt{\eta^{2}+\xi^{4}}+\xi^{2}}{2}}+i \operatorname{sign}(\eta) \sqrt{\frac{\sqrt{\eta^{2}+\xi^{4}}-\xi^{2}}{2}} \\
& =a+\sigma b i,
\end{aligned}
$$

where $\sigma=\operatorname{sign}(\eta)$.

We note here that, for fixed $0<x \leq 1$, the value of $\cosh (\tau x)$ in (10) is unbounded as $|\xi| \rightarrow \infty$. We can see that small errors in the data can blow up and completely destroy the solution for $0<x \leq 1$. Thus the Cauchy problem of $2 \mathrm{D}$ heat conduction equation is ill-posed. A feasible approach to stabilize the problem is to eliminate all high frequencies or to replace the "kernel" $\cosh (\tau x)$ by a bounded approximation kernel denoted by $k(x, \tau, \alpha)$, which has the following two common properties:

(I) If the parameter $\alpha$ is small, then for small $\tau$, the kernel $k(x, \tau, \alpha)$ is close to $\cosh (\tau x)$.

(II) If $\alpha$ is fixed, $k(x, \tau, \alpha)$ is bounded.

\section{Revised Tikhonov Regularization Method and Error Estimates}

To get a bounded approximation kernel $k(x, \tau, \alpha)$, a popular method is Tikhonov regularization, which is applied to solve the following minimization problem:

$$
\begin{aligned}
& \min F_{\alpha}(u)=\min \left\{\left\|K u(\cdot, y, t)-g^{\delta}(y, t)\right\|_{L^{2}\left(\mathbb{R}^{2}\right)}^{2}\right. \\
& \left.\quad+\alpha\|u(\cdot, y, t)\|_{L^{2}\left(\mathbb{R}^{2}\right)}^{2}\right\},
\end{aligned}
$$

where $\alpha$ is a regularization parameter and $K: u(\cdot, y, t) \rightarrow$ $g(y, t)$ is a linear bounded operator.

Lemma 2. There exists a unique solution to the above minimization problem. It is given by

$$
\begin{aligned}
& u_{\alpha}^{\delta}(x, y, t) \\
& \quad=\frac{1}{2 \pi} \int_{\mathbb{R}^{2}} \frac{\cosh (\tau x)}{1+\alpha|\cosh (\tau x)|^{2}} \widehat{g}^{\delta}(\xi, \eta) e^{i(\xi y+\eta t)} d \xi d \eta
\end{aligned}
$$


Proof. Let $I$ denote identity operator in $L^{2}$ and let $K^{*}$ be the adjoint of $K$. Then, the unique solution of the minimization problem (13) is given by

$$
u_{\alpha}^{\delta}=\left(\alpha I+K^{*} K\right)^{-1} K^{*} g^{\delta} .
$$

In order to obtain the explicit formula (14) from (15), we use Parseval's formula; one has

$$
\langle\widehat{K(x) u}, \widehat{v}\rangle=\langle K(x) u, v\rangle=\left\langle u, K^{*} v\right\rangle=\left\langle\widehat{u}, \widehat{K^{*} v}\right\rangle .
$$

According to $\widehat{K u}=(1 / \cosh (\tau x)) \widehat{u}(x, \xi, \eta)$, we find

$$
\widehat{K^{*} v}=\frac{1}{\overline{\cosh (\tau x)}} \widehat{v}(x, \xi, \eta) .
$$

Likewise,

$$
\widehat{K^{*} K u}=\frac{1}{|\cosh (\tau x)|^{2}} \widehat{u}(x, \xi, \eta) .
$$

Consequently,

$$
\left(\alpha+\frac{1}{|\cosh (\tau x)|^{2}}\right) \widehat{u}=\widehat{K^{*} g^{\delta}} .
$$

Using (17) and solving for $\widehat{u}_{\alpha}^{\delta}(x, \xi, \eta)$ in (19), we obtain

$$
\widehat{u}_{\alpha}^{\delta}(x, \xi, \eta)=\frac{\cosh (\tau x)}{1+\alpha|\cosh (\tau x)|^{2}} \widehat{g}^{\delta}(\xi, \eta) .
$$

Finally, (14) follows by an inverse Fourier transform.

From Lemma 2, we can see that the kernel function $k(x, \tau, \alpha)=\cosh (\tau x) /\left(1+\alpha|\cosh (\tau x)|^{2}\right)$ corresponds to the Tikhonov regularization method.

In the following, we use a much better filter $1 /(1+$ $\left.\alpha e^{2|\tau|}\right)$ to replace the original $1 /\left(1+\alpha|\cosh (\tau x)|^{2}\right)$; i.e., $k(x, \tau, \alpha)=\cosh (\tau x) /\left(1+\alpha e^{2|\tau|}\right)$. It corresponds to the regularized solution as follows:

$$
u_{\alpha}^{\delta}(x, y, t)=\frac{1}{2 \pi} \int_{\mathbb{R}^{2}} e^{i(\xi y+\eta t)} \frac{\cosh (\tau x)}{1+\alpha e^{2|\tau|}} \widehat{g}^{\delta}(\xi, \eta) d \xi d \eta,
$$

and is regarded as a revised Tikhonov regularization solution of $u(x, y, t)$ of problem 1 .

In the following, we give the convergence estimate for $\left\|u_{\alpha}^{\delta}(x, y, t)-u(x, y, t)\right\|$ by using an a priori choice rule for the regularization parameter. Before proceeding to derive the main result, we recall a proposition which we will use in the proof below.

Proposition 3 (see [14]). Let $0<x<2, \alpha>0$. We have the following inequality:

$$
\sup _{|\tau| \geq 0} \frac{e^{|\tau| x}}{1+\alpha e^{2|\tau|}} \leq\left(\frac{1}{\sqrt{\alpha}}\right)^{x} .
$$

Theorem 4. Suppose $u$ is the solution of problem 1 with the exact data $g$ and $u_{\alpha}^{\delta}$ is the regularized solution with the noise data $g^{\delta}$, and let $g^{\delta}$ satisfy (5) and the exact solution $u$ at $x=1$ satisfy (6). If $\alpha=\delta^{2} / E^{2}$ is selected, then for fixed $0<x<1$, one gets the error estimate

$$
\left\|u_{\alpha}^{\delta}(x, y, t)-u(x, y, t)\right\| \leq 3 \delta^{1-x} E^{x} .
$$

Proof. Using the Parseval's identity and Proposition 3, we have

$$
\begin{aligned}
& \left\|u_{\alpha}^{\delta}(x, y, t)-u_{\alpha}(x, y, t)\right\|=\left\|\hat{u}_{\alpha}^{\delta}(x, \xi, \eta)-\widehat{u}_{\alpha}(x, \xi, \eta)\right\| \\
& =\left(\int_{-\infty}^{\infty} \int_{-\infty}^{\infty}\left|\frac{\cosh (\tau x)}{1+\alpha e^{2|\tau|}} \widehat{g}^{\delta}(\xi, \eta)-\frac{\cosh (\tau x)}{1+\alpha e^{2|\tau|}} \widehat{g}(\xi, \eta)\right|^{2} d \xi d \eta\right)^{1 / 2} \\
& \leq \sup _{\xi, \eta \in \mathbb{R}}\left|\frac{\cosh (\tau x)}{1+\alpha e^{2|\tau|}}\right|\left\|\widehat{g}^{\delta}-\hat{g}\right\| \leq \sup _{\xi, \eta \in \mathbb{R}} \frac{e^{|\tau| x}}{1+\alpha e^{2|\tau|}} \delta \leq\left(\frac{1}{\sqrt{\alpha}}\right)^{x} \delta, \\
& \left\|u_{\alpha}(x, y, t)-u(x, y, t)\right\|=\left\|\widehat{u}_{\alpha}(x, \xi, \eta)-\widehat{u}(x, \xi, \eta)\right\| \\
& =\left(\int_{-\infty}^{\infty} \int_{-\infty}^{\infty}\left|\frac{\cosh (\tau x)}{1+\alpha e^{2|\tau|}} \widehat{g}-\cosh (\tau x) \hat{g}\right|^{2} d \xi d \eta\right)^{1 / 2} \\
& =\left(\int_{-\infty}^{\infty} \int_{-\infty}^{\infty}\left|\frac{\alpha \cosh (\tau x) e^{2|\tau|}}{1+\alpha e^{2|\tau|}} \hat{g}\right|^{2} d \xi d \eta\right)^{1 / 2} \\
& =\left(\int_{-\infty}^{\infty} \int_{-\infty}^{\infty}\left|\frac{\alpha \cosh (\tau x) e^{2|\tau|}}{1+\alpha e^{2|\tau|}} \frac{1}{\cosh (\tau)} \widehat{u}(1, \xi, \eta)\right|^{2} d \xi d \eta\right)^{1 / 2} \\
& \leq \alpha \sup _{\xi, \eta \in \mathbb{R}} \frac{e^{2|\tau|}}{1+\alpha e^{2|\tau|}}\left|\frac{\cosh (\tau x)}{\cosh (\tau)}\right|\|\widehat{u}(1, \cdot, \cdot)\| \\
& \leq \alpha \sup _{\xi, \eta \in \mathbb{R}} \frac{e^{2|\tau|}}{1+\alpha e^{2|\tau|}} 2 e^{-(1-x)|\tau|}\|\widehat{u}(1, \cdot, \cdot)\| \leq 2 \alpha\left(\frac{1}{\sqrt{\alpha}}\right)^{1+x} E .
\end{aligned}
$$

Combining (24), (25), and the condition $\alpha=\delta^{2} / E^{2}$, we have

$$
\left\|u_{\alpha}^{\delta}(x, y, t)-u(x, y, t)\right\| \leq 3 \delta^{1-x} E^{x} .
$$

Remark 5. Compared with [11, 12], where the convergence estimate is of logarithmic type, the convergence estimate we get is of Hölder type.

Remark 6. In Theorem 4, we do not obtain the error estimate at $x=1$; in order to obtain that, we should use a stronger priori assumption as follows:

$$
\|u(1, \cdot, \cdot)\|_{p} \leq E
$$

where $\|\cdot\|_{p}$ denotes the norm on Sobolev space $H^{p}\left(\mathbb{R}^{2}\right)$ defined by

$$
\begin{gathered}
\|u(1, \cdot, \cdot)\|_{p}=\left(\int_{-\infty}^{\infty} \int_{-\infty}^{\infty}\left(1+|\xi|^{2}+|\eta|^{2}\right)^{p}\right. \\
\left.\cdot|\widehat{u}(1, \xi, \eta)|^{2} d \xi d \eta\right)^{1 / 2}, \quad p>0 .
\end{gathered}
$$


Theorem 7. Suppose $u(1, y, t)$ is the solution of problem 1 with the exact data $g$ and $u_{\alpha}^{\delta}(1, y, t)$ is the regularized solution with the noise data $g^{\delta}$, and let $g^{\delta}$ satisfy (5) and the exact solution $u$ at $x=1$ satisfy (27). If $\alpha=\delta / E$ is selected, then for fixed $x=1$, one gets the error estimate

$$
\begin{aligned}
& \left\|u_{\alpha}^{\delta}(1, y, t)-u(1, y, t)\right\| \\
& \quad \leq \delta^{1 / 2} E^{1 / 2}+E \max \left\{\left(\frac{\delta}{E}\right)^{1 / 2},\left(\frac{1}{8} \ln \frac{E}{\delta}\right)^{-p}\right\} .
\end{aligned}
$$

Proof. Similar to the proof of Theorem 4, we know that

$$
\begin{aligned}
\left\|u_{\alpha}^{\delta}(1, y, t)-u_{\alpha}(1, y, t)\right\| & \leq \frac{\delta}{\sqrt{\alpha}} \\
\left\|u_{\alpha}(1, y, t)-u(1, y, t)\right\| & =\left\|\widehat{u}_{\alpha}(1, \xi, \eta)-\widehat{u}(1, \xi, \eta)\right\|=\left(\int_{-\infty}^{\infty} \int_{-\infty}^{\infty}\left|\frac{\cosh (\tau)}{1+\alpha e^{2|\tau|}} \widehat{g}-\cosh (\tau) \hat{g}\right|^{2} d \xi d \eta\right)^{1 / 2} \\
& =\left(\int_{-\infty}^{\infty} \int_{-\infty}^{\infty}\left|\frac{\alpha \cosh (\tau) e^{2|\tau|}}{1+\alpha e^{2|\tau|}} \hat{g}\right|^{2} d \xi d \eta\right)^{1 / 2} \\
& =\left(\int_{-\infty}^{\infty} \int_{-\infty}^{\infty}\left|\frac{\alpha e^{2|\tau|}}{1+\alpha e^{2|\tau|}}\left(1+|\xi|^{2}+|\eta|^{2}\right)^{-p / 2}\left(1+|\xi|^{2}+|\eta|^{2}\right)^{p / 2} \cosh (\tau) \hat{g}\right|^{2} d \xi d \eta\right)^{1 / 2} \\
& \leq\left(\sup _{\xi, \eta \in \mathbb{R}} \frac{\alpha e^{2|\tau|}}{1+\alpha e^{2|\tau|}}\left(1+|\xi|^{2}+|\eta|^{2}\right)^{-p / 2}\right)\|\widehat{u}(1, \cdot, \cdot)\|_{p} .
\end{aligned}
$$

Now, we distinguish two cases to estimate (31).

Case 1. For $\sqrt{|\eta|}+|\xi| \leq(1 / 2) \ln (1 / \sqrt{\alpha})$, we have

$$
|\tau|=\left|\sqrt{\left(i \eta+\xi^{2}\right)}\right| \leq \sqrt{|\eta|}+|\xi| .
$$

Therefore, we can derive that

$$
\begin{aligned}
& \frac{\alpha e^{2|\tau|}}{1+\alpha e^{2|\tau|}}\left(1+|\xi|^{2}+|\eta|^{2}\right)^{-p / 2} \leq \alpha e^{2|\tau|} \leq \alpha e^{2(\sqrt{|\eta|}+|\xi|)} \\
& \quad \leq \sqrt{\alpha} .
\end{aligned}
$$

We can see that it goes to zero as $\alpha \rightarrow 0$.

Case 2. For $\sqrt{|\eta|}+|\xi| \geq(1 / 2) \ln (1 / \sqrt{\alpha})$, we have

$$
\begin{gathered}
\frac{\alpha e^{2|\tau|}}{1+\alpha e^{2|\tau|}}\left(1+|\xi|^{2}+|\eta|^{2}\right)^{-p / 2} \\
\leq\left(1+|\xi|^{2}+|\eta|^{2}\right)^{-p / 2} .
\end{gathered}
$$

If $\sqrt{|\eta|} \geq|\xi| \geq 0$, we have

$$
2 \sqrt{|\eta|} \geq \sqrt{|\eta|}+|\xi| \geq \frac{1}{2} \ln \frac{1}{\sqrt{\alpha}}
$$

i.e., $\sqrt{|\eta|} \geq(1 / 4) \ln (1 / \sqrt{\alpha})$. Therefore,

$$
\begin{aligned}
\left(1+|\xi|^{2}+|\eta|^{2}\right)^{-p / 2} & \leq\left(|\xi|^{2}+|\eta|^{2}\right)^{-p / 2} \leq|\eta|^{-p} \\
& =(\sqrt{|\eta|})^{-2 p} \leq\left(\frac{1}{4} \ln \frac{1}{\sqrt{\alpha}}\right)^{-2 p} .
\end{aligned}
$$

Now using the triangle inequality and combining (30) and (39), we can easily get the convergence estimate

$$
\begin{aligned}
\left\|u_{\alpha}^{\delta}(1, y, t)-u(1, y, t)\right\| \\
\leq\left\|\widehat{u}_{\alpha}^{\delta}(1, \xi, \eta)-\widehat{u}_{\alpha}(1, \xi, \eta)\right\| \\
\quad+\left\|\widehat{u}_{\alpha}(1, \xi, \eta)-\widehat{u}(1, \xi, \eta)\right\| \\
\leq \delta^{1 / 2} E^{1 / 2}+E \max \left\{\left(\frac{\delta}{E}\right)^{1 / 2},\left(\frac{1}{8} \ln \frac{E}{\delta}\right)^{-p}\right\} .
\end{aligned}
$$

Combining (31), (33), (36), and (38), we obtain

$$
\begin{aligned}
& \left\|u_{\alpha}(1, y, t)-u(1, y, t)\right\| \\
& \leq E \max \left\{\sqrt{\alpha},\left(\frac{1}{4} \ln \frac{1}{\sqrt{\alpha}}\right)^{-p}\right\} .
\end{aligned}
$$

i.e., $|\xi| \geq(1 / 4) \ln (1 / \sqrt{\alpha})$. Therefore,

$$
\begin{aligned}
\left(1+|\xi|^{2}+|\eta|^{2}\right)^{-p / 2} & \leq\left(|\xi|^{2}+|\eta|^{2}\right)^{-p / 2} \leq|\xi|^{-p} \\
& \leq\left(\frac{1}{4} \ln \frac{1}{\sqrt{\alpha}}\right)^{-p} .
\end{aligned}
$$

If $0 \leq \sqrt{|\eta|} \leq|\xi|$, we have

$$
2|\xi| \geq \sqrt{|\eta|}+|\xi| \geq \frac{1}{2} \ln \frac{1}{\sqrt{\alpha}}
$$

(1) 


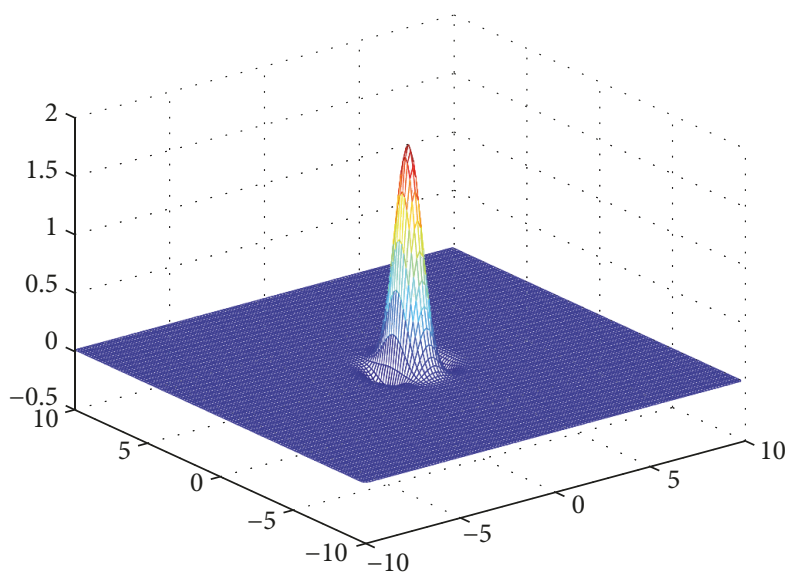

(a)

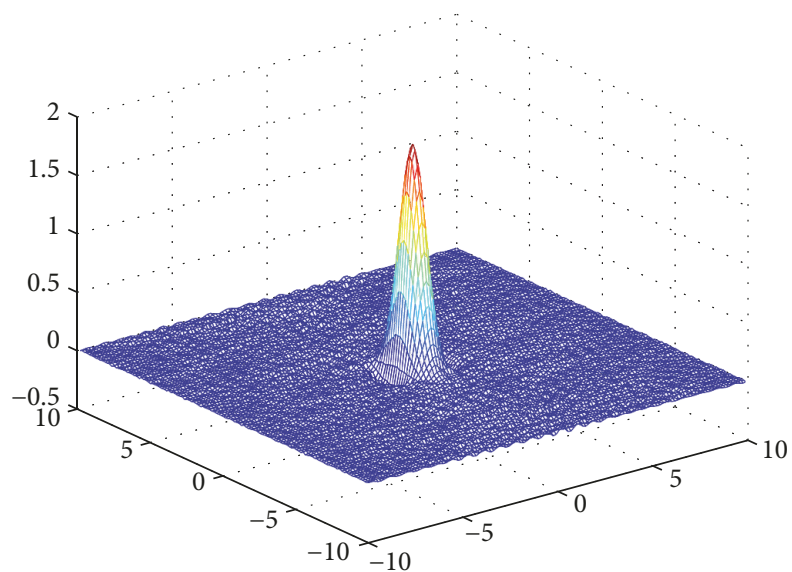

(c)

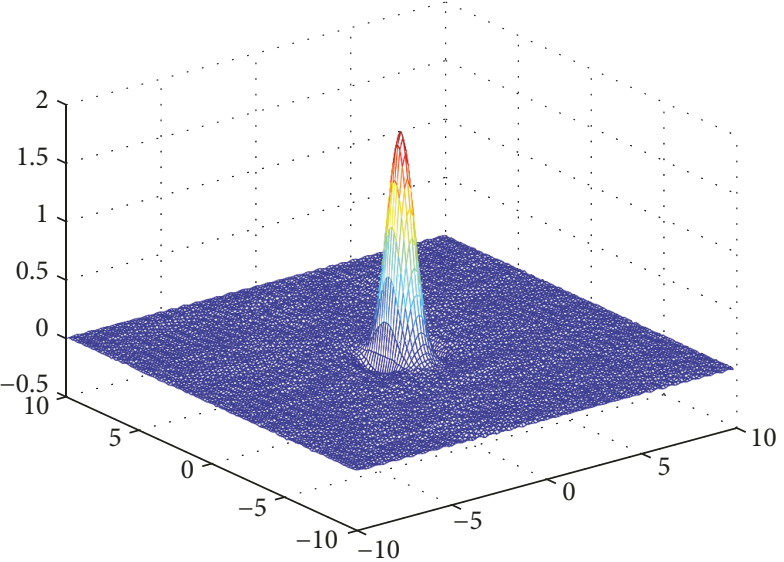

(b)

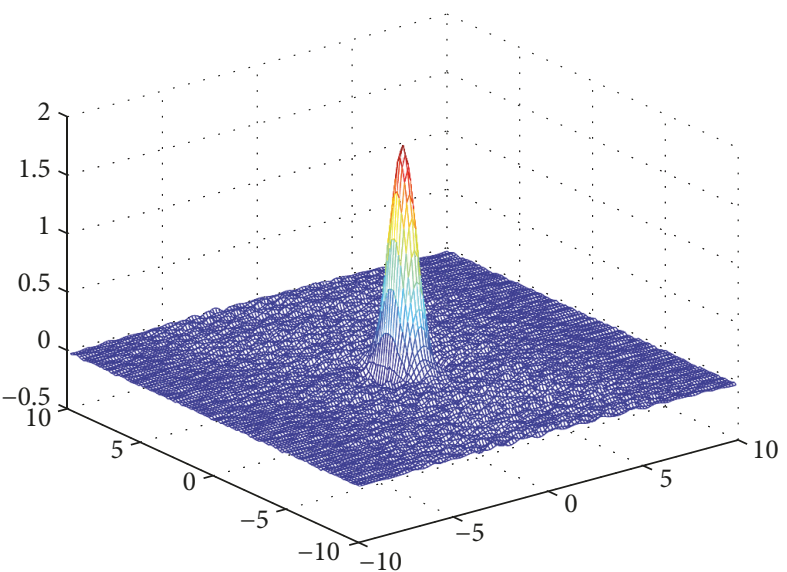

(d)

FIGURE 1: The exact solution and its approximation solution at $x=0.8$ : (a) the exact solution, (b) its approximation solution with $\varepsilon=0.0001$, (c) its approximation solution with $\varepsilon=0.001$, (d) its approximation solution with $\varepsilon=0.01$.

\section{Numerical Aspect}

In the section, we present a numerical example intended to illustrate the behaviour of the proposed method.

The numerical example is constructed in the following way: first we select the initial data $u(0, y, t)=g(y, t)$ and $u_{x}(0, y, t)=0$. Then we added a random distributed perturbation to the data function obtaining vector $g^{\delta}(y, t)$; i.e.,

$$
g^{\delta}(y, t)=g(y, t)+\varepsilon \operatorname{randn}(\operatorname{size}(g(y, t))),
$$

where

$$
\begin{aligned}
g(y, t) & =\left(g\left(y_{1}, t_{1}\right), g\left(y_{2}, t_{2}\right), \ldots, g\left(y_{n}, t_{n}\right)\right)^{T}, \\
y_{i} & =-10+(i-1) \Delta y, \\
\Delta y & =\frac{20}{n-1}, i=1,2, \ldots, n, \\
t_{j} & =-10+(j-1) \Delta t, \\
\Delta t & =\frac{20}{n-1}, j=1,2, \ldots, n .
\end{aligned}
$$

Then the total noise $\delta$ can be measured in the sense of Root Mean Square Error according to

$$
\begin{aligned}
\delta & :=\left\|g^{\delta}(y, t)-g(y, t)\right\|_{l^{2}} \\
& =\sqrt{\frac{1}{n^{2}} \sum_{i=1}^{n} \sum_{j=1}^{n}\left(g_{i}^{\delta}\left(y_{i}, t_{j}\right)-g_{i}\left(y_{i}, t_{j}\right)\right)^{2}} .
\end{aligned}
$$

The function "randn $(\cdot)$ " generates arrays of random numbers whose elements are normally distributed with mean 0 , variance $\sigma^{2}=1$, and standard deviation $\sigma=1$; "randn(size $(g(y, t)))$ " returns array of random entries that is of the same size as $g(y, t)$.

\section{Example 8. We choose}

$$
g(y, t)=e^{-y^{2}-t^{2}} .
$$

Our tests about this example correspond to Figures 1-3. The numerical results for $u$ and $u_{\alpha}^{\delta}$ with different error level 


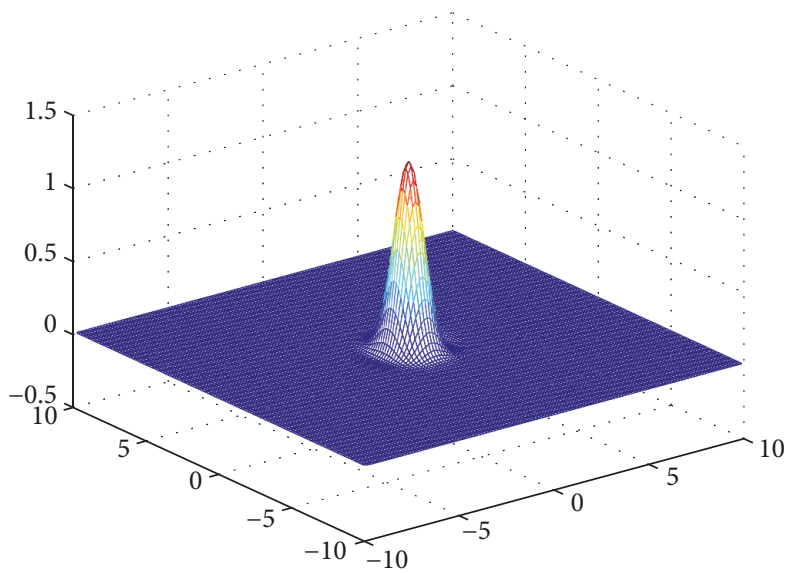

(a)

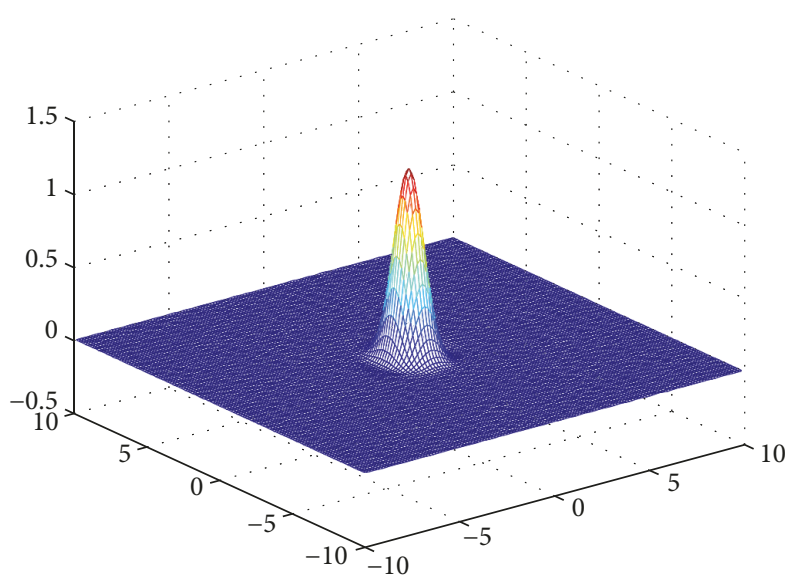

(c)

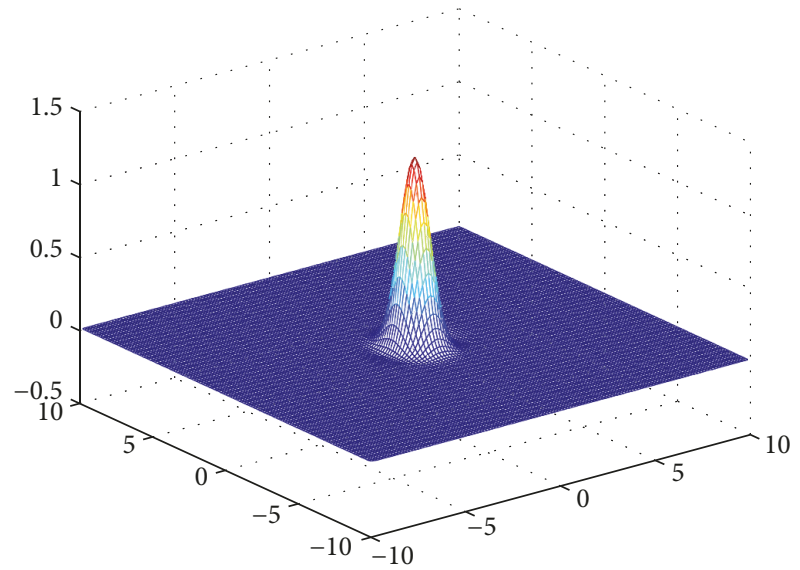

(b)

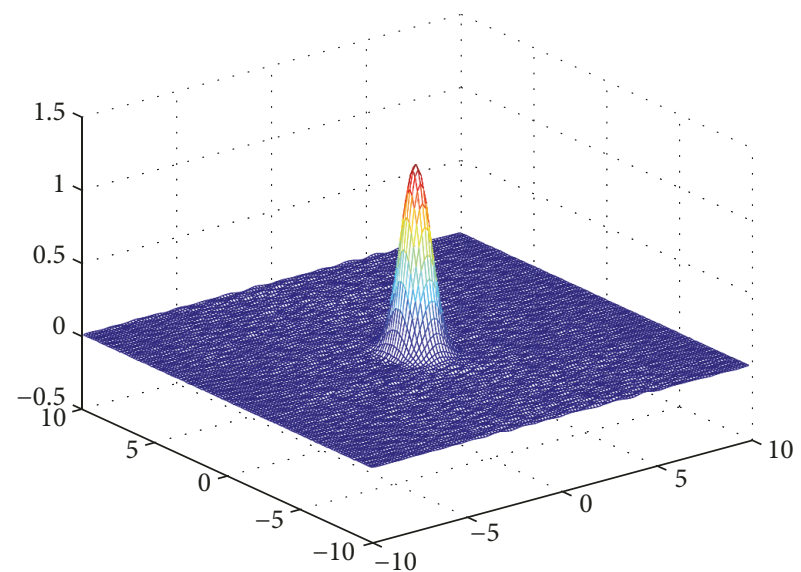

(d)

Figure 2: The exact solution and its approximation solution at $x=0.5$ : (a) the exact solution, (b) its approximation solution with $\varepsilon=0.0001$, (c) its approximation solution with $\varepsilon=0.001$, (d) its approximation solution with $\varepsilon=0.01$.

$\varepsilon=0.0001,0.001,0.01$ at different point $x=0.8,0.5$ are shown in Figures 1-2. The numerical result for $u$ and $u_{\alpha}^{\delta}$ with error level $\varepsilon=0.01$ at different point $x=0.9,0.6,0.1$ is shown in Figure 3.

From Figures 1-2, we can see that the smaller the $\varepsilon$ is, the better the computed solution is. Figure 3 shows that the numerical result becomes worse when $x$ approaches 1 .

\section{Conclusion}

In this paper, the Cauchy problem of $2 \mathrm{D}$ heat conduction equation is considered. We regularize it by a revised Tikhonov regularization method for overcoming its ill-posedness. The error estimate is obtained under an a priori regularization parameter choice rule. A numerical example shows that our proposed method is effective and stable.

\section{Conflicts of Interest}

The authors declare that they have no conflicts of interest.

\section{Authors' Contributions}

The authors contributed equally to the writing of this paper. All authors read and approved the final manuscript.

\section{Acknowledgments}

The research of Songshu Liu was supported by a grant from the National Science Foundation of Hebei Province (A2017501021), the Fundamental Research Funds for the Central Universities (N162303007), and the PhD Foundation for Northeastern University at Qinhuangdao (XNB201612). 


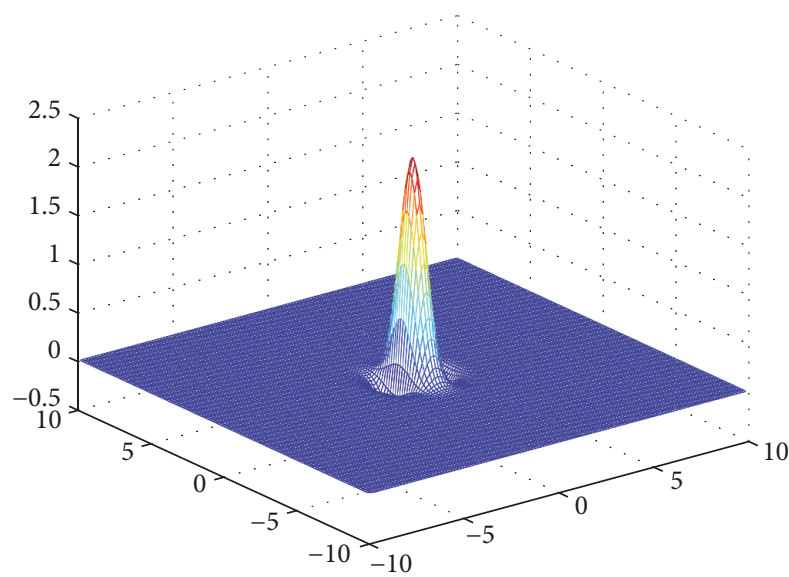

(a)

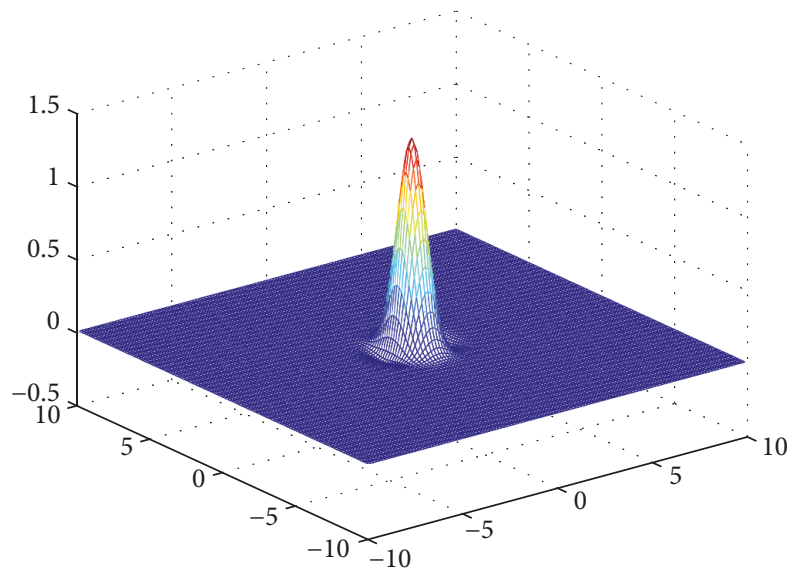

(c)

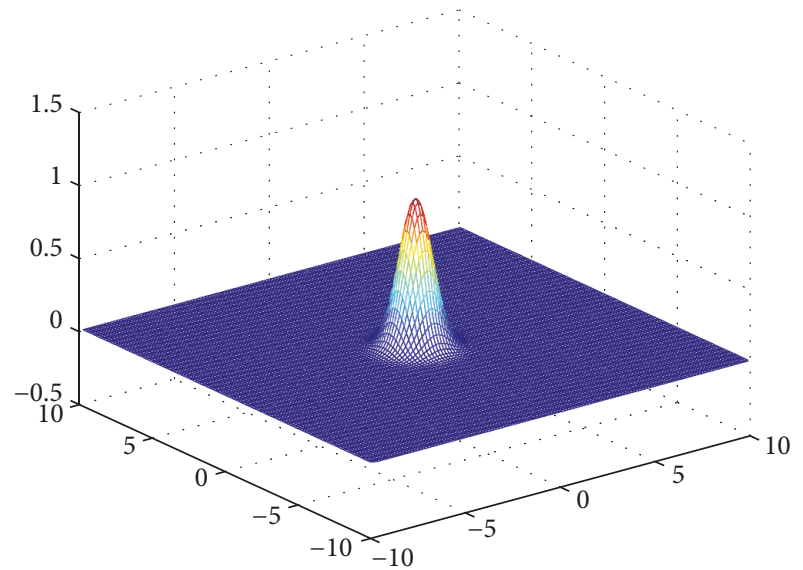

(e)

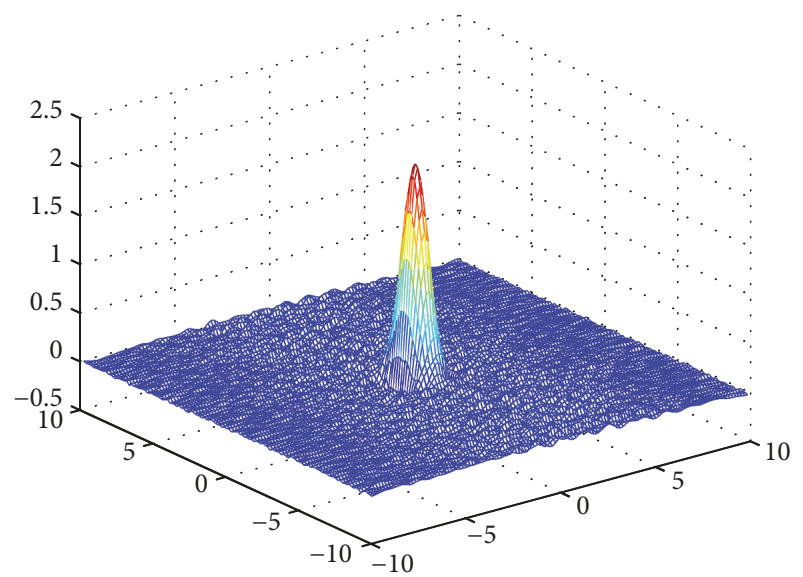

(b)

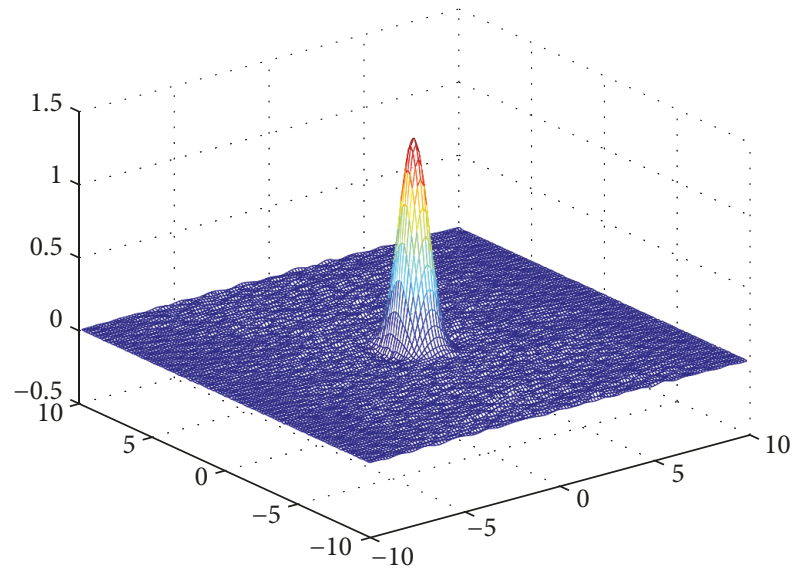

(d)

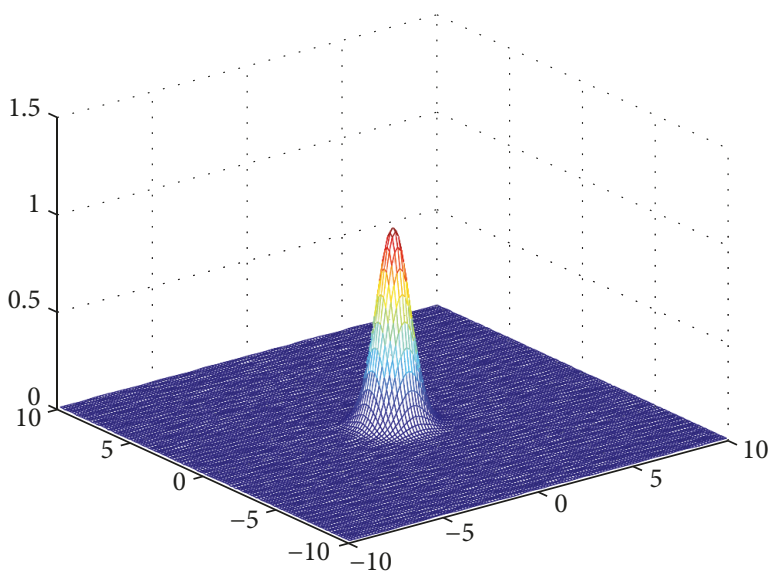

(f)

FIGURE 3: The exact solution and its approximation solution: (a) the exact solution at $x=0.9$, (b) its approximation solution with $\varepsilon=0.01$ at $x=0.9$, (c) the exact solution at $x=0.6$, (d) its approximation solution with $\varepsilon=0.01$ at $x=0.6$, (e) the exact solution at $x=0.1$, (f) its approximation solution with $\varepsilon=0.01$ at $x=0.1$. 
The research of Lixin Feng was supported by a grant from the New Century Foundation of Heilongjiang Province (1253-NECT-019), Science and Technology Innovation Team in Higher Education Institutions of Heilongjiang Province (2014TD005).

\section{References}

[1] J. K. Beck, B. Blackwell, and S. R. Clair, SR: Inverse Heat Conduction, Ill-posed Problems, Wiley, New York, USA, 1985.

[2] F. Berntsson, "A spectral method for solving the sideways heat equation," Inverse Problems, vol. 15, no. 4, pp. 891-906, 1999.

[3] J. R. Cannon, "A Cauchy problem for the heat equation," Annali di Matematica Pura ed Applicata. Serie Quarta, vol. 66, pp. 155165, 1964.

[4] P. Manselli and K. Miller, "Calculation of the surface temperature and heat flux on one side of a wall from measurements on the opposite side," Annali di Matematica Pura ed Applicata. Serie Quarta, vol. 123, pp. 161-183, 1980.

[5] L. Elden, "Numerical solution of the sideways heat equation by difference approximation in time," Inverse Problems, vol. 11, no. 4, pp. 913-923, 1995.

[6] L. Eldén, F. Berntsson, and T. Regin'ska, "Wavelet and Fourier methods for solving the sideways heat equation," SIAM Journal on Scientific Computing, vol. 21, no. 6, pp. 2187-2205, 2000.

[7] L. Eldén, "Hyperbolic approximations for a Cauchy problem for the heat equation," Inverse Problems, vol. 4, no. 1, pp. 59-70, 1988.

[8] L. Eldén, "Approximations for a Cauchy problem for the heat equation," Inverse Problems, vol. 3, no. 2, pp. 263-273, 1987.

[9] P. Jonas and A. K. Louis, "Approximate inverse for a onedimensional inverse heat conduction problem," Inverse Problems, vol. 16, no. 1, pp. 175-185, 2000.

[10] T. I. Seidman and L. Eldén, "An "optimal filtering" method for the sideways heat equation," Inverse Problems, vol. 6, no. 4, pp. 681-696, 1990.

[11] Z. Qian and C.-L. Fu, "Regularization strategies for a two-dimensional inverse heat conduction problem," Inverse Problems, vol. 23, no. 3, pp. 1053-1068, 2007.

[12] J. Li and F. Wang, "Simplified Tikhonov regularization for two kinds of parabolic equations," Journal of the Korean Mathematical Society, vol. 48, no. 2, pp. 311-327, 2011.

[13] J. Zhao, S. Liu, and T. Liu, "A modified kernel method for solving Cauchy problem of two-dimensional heat conduction equation," Advances in Applied Mathematics and Mechanics, vol. 7, no. 1, pp. 31-42, 2015.

[14] A. Carasso, "Determining surface temperatures from interior observations," SIAM Journal on Applied Mathematics, vol. 42, no. 3, pp. 558-574, 1982.

[15] C.-L. Fu, "Simplified Tikhonov and Fourier regularization methods on a general sideways parabolic equation," Journal of Computational and Applied Mathematics, vol. 167, no. 2, pp. 449-463, 2004.

[16] F. Yang and C.-L. Fu, "The method of simplified Tikhonov regularization for dealing with the inverse time-dependent heat source problem," Computers and Mathematics with Applications. An International Journal, vol. 60, no. 5, pp. 1228-1236, 2010.

[17] F. Yang and C.-L. Fu, "A simplified Tikhonov regularization method for determining the heat source," Applied Mathematical
Modelling: Simulation and Computation for Engineering and Environmental Systems, vol. 34, no. 11, pp. 3286-3299, 2010.

[18] J. Zhao, S. Liu, and T. Liu, "An inverse problem for spacefractional backward diffusion problem," Mathematical Methods in the Applied Sciences, vol. 37, no. 8, pp. 1147-1158, 2014. 


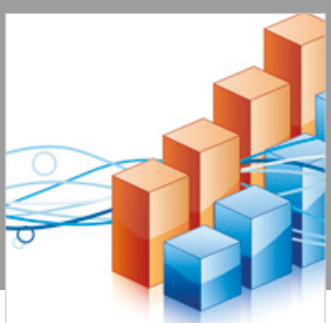

Advances in

Operations Research

\section{-n-m}
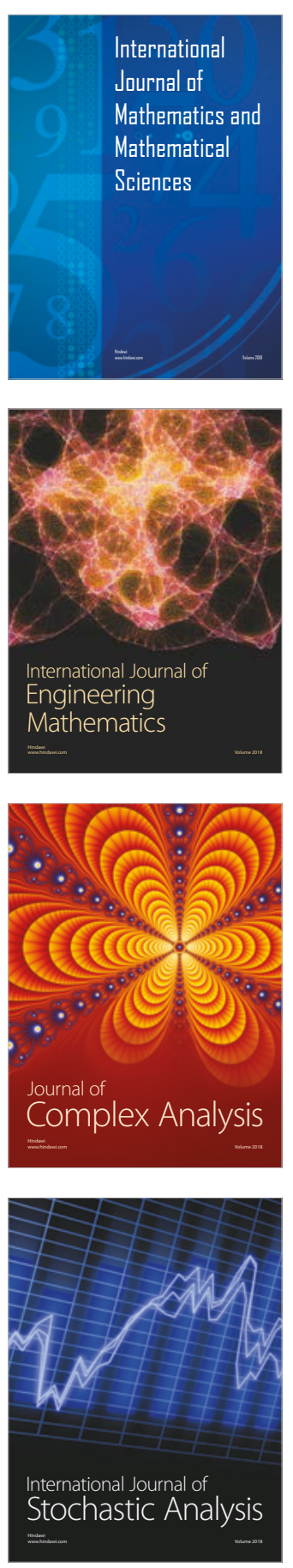
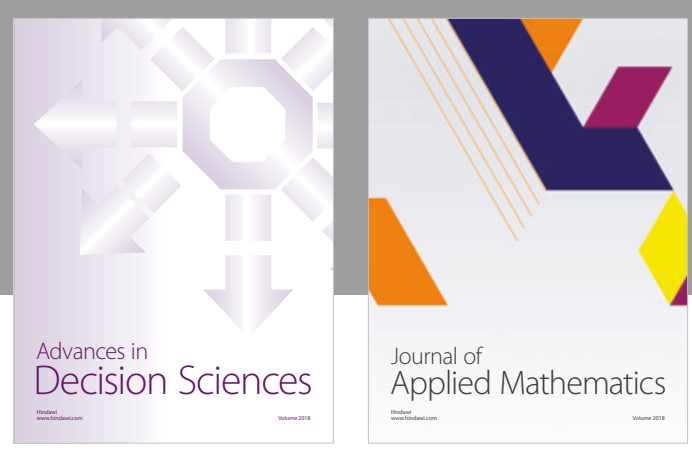

Journal of

Applied Mathematics
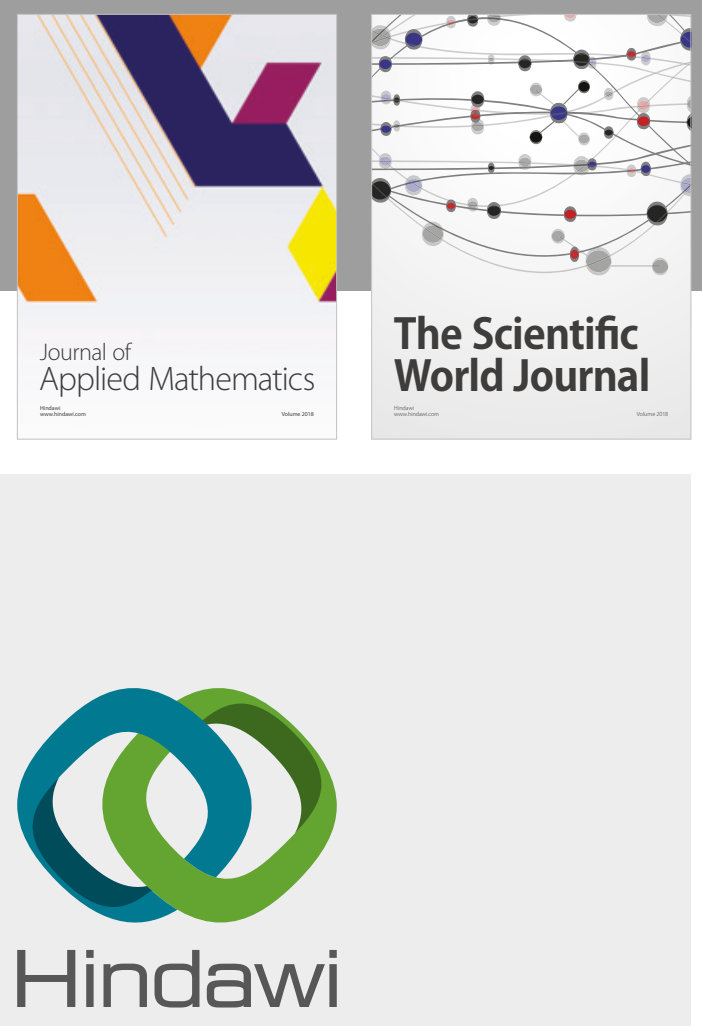

Submit your manuscripts at

www.hindawi.com

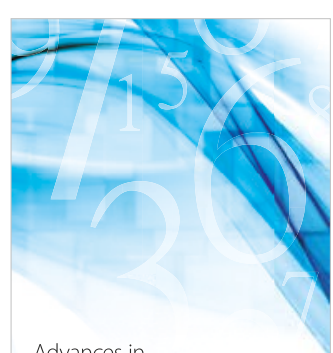

Advances in
Numerical Analysis
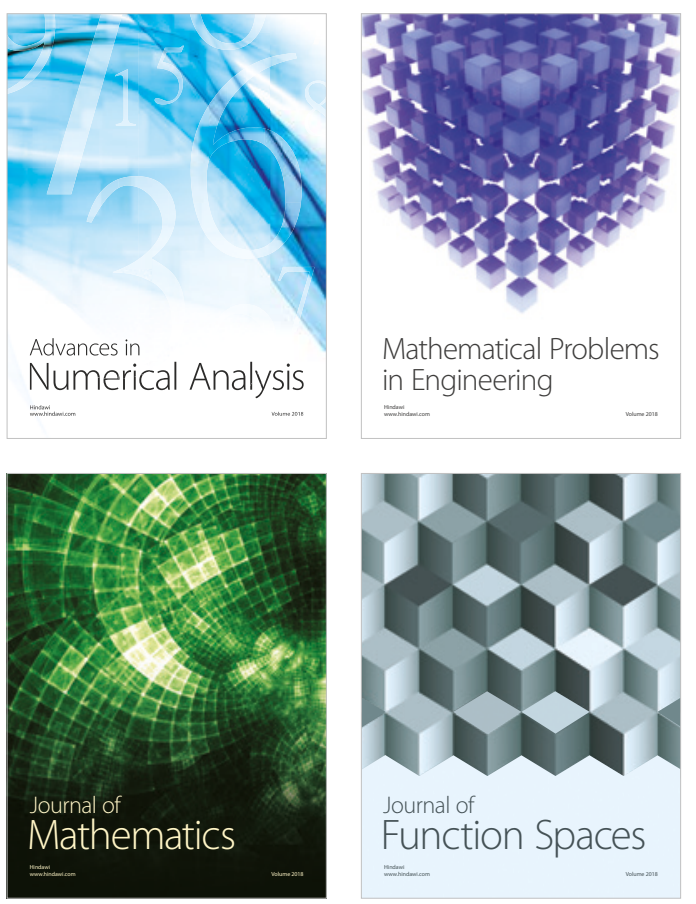

Mathematical Problems in Engineering

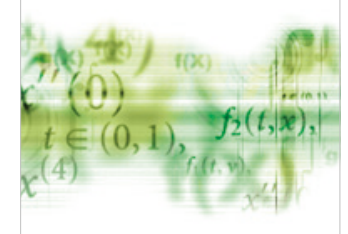

International Journal of

Differential Equations

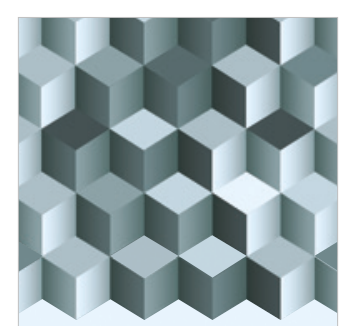

Journal of

Function Spaces

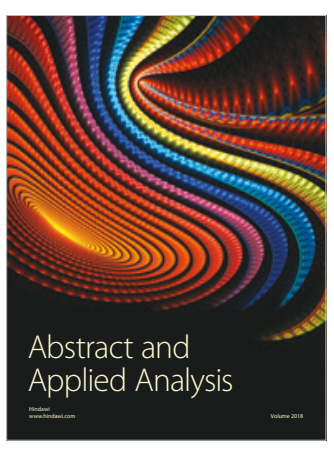

The Scientific

World Journal

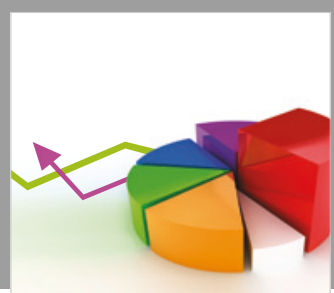

Journal of

Probability and Statistics
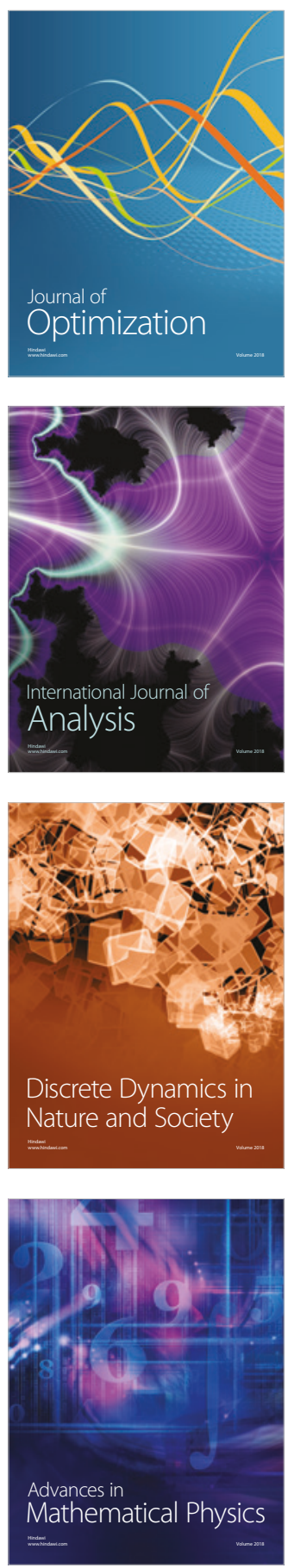